\title{
nipi Delta-Doping Superlattices for Amplitude Modulation
}

\author{
C. V.-B. Tribuzy, S. M. Landi, M. P. Pires, R. Butendeich; P. L. Souza, \\ Laboratório de Semicondutores, Centro de Estudos em Telecomunicaçôes, \\ Pontifícia Universidade Católica do Rio de Janeiro, \\ Rua Marquês de São Vicente 225, Rio de Janeiro, 22453-900, Brazil
}

A. C. Bittencourt, G. E. Marques, Instituto de Física, Universidade Federal de São Carlos, São Carlos, 13565-905 Brazil and A. B. Henriques

Instituto de Física, Universidade de São Paulo, São Paulo 05315-970, Brazil

Received on April 23, 2001

\begin{abstract}
GaAs/AlGaAs multiple quantum well structures containing an nipi delta-doping superlattice, where the $n$-type doping is inserted in the quantum wells and the $p$-type in the barriers, have been studied in detail to evaluate their potential for use in the fabrication of amplitude modulators. It is shown that $\mathrm{C}$ is an adequate $p$-type dopant for such structures, however, little flexibility is found in the growth conditions, in particular for the $\mathrm{V}$ to III fluxes ratio, for obtaining such layers. It is also observed that the required balance between $n$ and $p$ type doping levels is not trivial to be achieved due to the presence of interface hole traps whose population depends on the quantum well doping concentration. In addition, the observed photoluminescence near-edge emission at room temperature occurs at essentially the same energy as that of an equivalent undoped structure. Finally, no deep level emissions are observed which could deteriorate the device performance.
\end{abstract}

\section{Introduction}

Recent demands for multiterabit communication require external amplitude modulators operating at low voltages. Amplitude modulators based on the Quantum Confined Stark Effect (QCSE) in III-V semiconductors multiple quantum well (MQW) systems are suitable for meeting these technological demands and therefore, much attention has been devoted to their development $t^{1,2}$. One of the crucial requirements for efficient modulation at high bit rates is that the change in absorption per applied voltage be as large as possible. In other words, the Stark shift should be maximized. The larger the quantum well is, the larger the Stark shift ${ }^{3}$. However, increasing the quantum well width decreases the oscillator strength for absorption. Thus, a compromise is imposed. An alternative for increasing the Stark shift has been proposed by Batty and Allsopp ${ }^{4}$. They have theoretically shown that the introduction of an nipi delta-doping superlattice in a MQW structure, where the quantum well is $n$-deltadoped while the barrier is $p$-delta-doped, may double the Stark shift.

If the nipi-MQW structures are expected to be used in amplitude modulators some requirements should be fulfiled. The presence of the nipi delta-doping superlattice should not introduce energy levels in the forbidden gap, otherwise, in the ON state of the device, light could be absorbed, dramatically increasing insertion losses. For applications in amplitude modulators, where the MQWs form the active region of the device, it is crucial that the net doping corresponds to an undoped structure, so that the applied electric field is uniformly distributed over the entire MQW region. It is therefore essential to balance out the electron and the hole concentrations in the delta layers. In this article, first, results of a thorough investigation of $\mathrm{C}$ deltadoping of AlGaAs grown by MOVPE using $\mathrm{CBr}_{4}$ as the $\mathrm{C}$ source are presented. Much attention is devoted to the changes in doping level as a function of the $\mathrm{V}$ to III fluxes ratio, V/III. It is experimentally shown that only within a small range of this ratio it is possible to obtain $\mathrm{C}$ delta-doped AlGaAs layers with all

\footnotetext{
${ }^{*}$ On leave from the Physikalisches Institut, University of Stuttgart, Germany.
} 
the properties required for the fabrication of amplitude modulators. Second, it is shown that in GaAs/AlGaAs nipi-MQW structures the fundamental optical transition is basically not shifted with respect to that of the undoped structure and no optical transition below the fundamental gap at room temperature is observed. Finally, transport measurements demonstrate that the $p$ doping efficiency changes when MQWs are grown with $n$ and $p$ dopants in the QWs and in the barriers, respectively. This renders the balance of $n$ and $p$ doping more delicate to be achieved.

\section{Experimental details}

All samples were grown by metalorganic vapor phase epitaxy on an AIX 200 reactor at $630^{\circ} \mathrm{C}$ and 100 mbar. TMGa, TMAl, $\mathrm{AsH}_{3}, \mathrm{SiH}_{4}$ and $\mathrm{CBr}_{4}$ were used as $\mathrm{Ga}$, $\mathrm{Al}, \mathrm{As}, \mathrm{Si}$ and $\mathrm{C}$ sources, respectively. For the $\mathrm{C}$ doped layers, on a $\mathrm{Cr}$-doped (100) oriented GaAs substrate, after a $500 \AA$ thick undoped GaAs buffer layer a 2000 $\AA$ thick AlGaAs layer homogeneously doped with $\mathrm{C}$ was deposited. The $\mathrm{Al}$ content in the alloy is $28 \%$, as determined by $\mathrm{x}$-ray measurements. In the case of the delta-doped samples, $\mathrm{C}$ was introduced during growth according to the following sequence. After a $1000 \AA$ thick undoped AlGaAs layer was grown, the TMAl and TMGa were switched off for 15 seconds while the $\mathrm{AsH}_{3}$ flux was switched from a high to a low value. Then the $\mathrm{Ga}, \mathrm{Al}$ and $\mathrm{C}$ sources were switched on for 2.5 seconds. After this short period these sources were switched off for 10 seconds while the $\mathrm{AsH}_{3}$ flux was raised to the original high value. At this point, the TMAl and TMGa were turned on and another $1000 \AA$ thick undoped AlGaAs layer was grown. The arsine flux should be such that minimizes $\mathrm{C}$ incorporation during growth of the undoped layer and optimizes it in the doped layer. Thus the $\mathrm{AsH}_{3}$ flux has to be changed during the growth of the delta-doped samples. Various V/III and $\mathrm{CBr}_{4}$ fluxes were used for the growth of the samples. Before removing the samples from the reactor chamber they were all annealed at $600^{\circ} \mathrm{C}$ for 15 minutes in $\mathrm{N}_{2}$ for removal of $\mathrm{H}$ atoms. For the GaAs/AlGaAs MQW samples, a $300 \AA$ GaAs buffer layer was first grown followed by a thick AlGaAs layer which was delta-doped $25 \AA$ before the growth of the MQW layers. The MQW structure consists of 20 periods of $100 \AA$ thick GaAs QWs, $n$-delta-doped using Si in the center, and $50 \AA$ thick AlGaAs barriers, C deltadoped in the center. The last barrier is $500 \AA$ thick and its $\mathrm{C}$ delta layer was introduced $25 \AA$ from the last grown QW. Growth interruption was used to produce the Si delta-doped GaAs layers.

The current carrier concentration and mobility were determined by Hall effect measurements at room tem- perature (RT) and at $77 \mathrm{~K}$ using an HL 5500 equipment. The capacitance vs. voltage $(\mathrm{C}-\mathrm{V})$ profiles were obtained by the electrochemical profiler PN 4300 to determine the delta layers localization both in GaAs and AlGaAs. The photoluminescence (PL) measurements were carried out with the $514 \mathrm{~nm}$ line of an $\mathrm{Ar}^{+}$laser for excitation. The signal was dispersed by a $250 \mathrm{~mm}$ monochromator and detected by a Ge nitrogen cooled photodetector. PL experiments were performed for temperatures between 20 and $300 \mathrm{~K}$.

\section{Results and discussion}

First, the difficulties and results involving $\mathrm{C}$ deltadoped bulk AlGaAs layers will be addressed, followed by the results of $\mathrm{Si}$ delta-doped GaAs bulk layers. Then, results on GaAs/AlGaAs MQW containing either $n$ or $p$ delta-doped layers centered in the QW and in the barrier, respectively, will be presented and discussed. Finally, the results obtained with the nipiMQW samples will be reported and analysed.

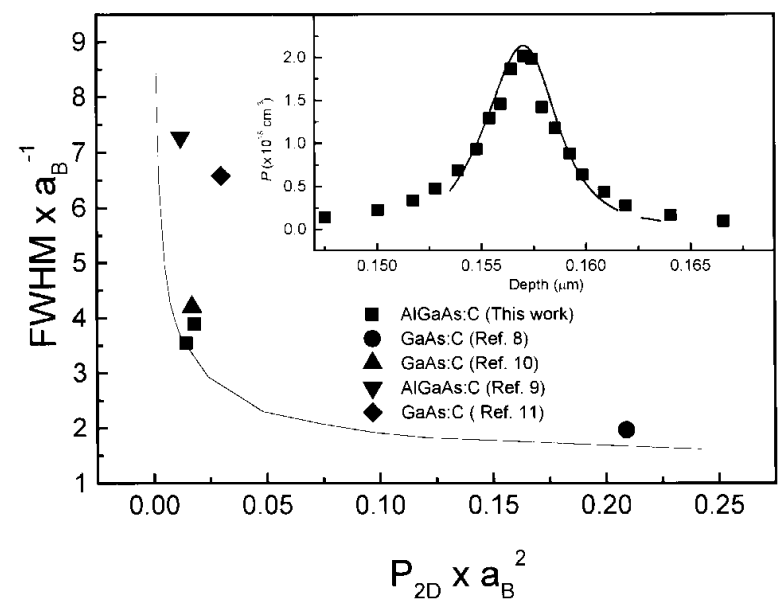

Figure 1. Full width at half maximum (FWHM) of the capacitance versus voltage profiles $(\mathrm{CV})$ in units of the Bohr radius $\left(\mathrm{a}_{B}=11.4 \AA\right)$ as a function of the areal net hole concentration, $\mathrm{P}_{2 D}$, in units of $\mathrm{a}_{B}^{-2}$. The solid line corresponds to the theoretical calculations, the squares refer to the experimental results of this work while the other data points correspond to data from the literature as specified by the labels. The inset shows the CV profile for one sample, where the solid line is the simulation for an impurity confinement equivalent to a gaussian of $5 \AA$ width while the scattered points correspond to the experimental data.

The solid dots in the inset of Fig. 1 represent the net hole concentration, $[\mathrm{P}]$, for one typical $\mathrm{C}$ deltadoped AlGaAs sample obtained by CV measurements. The CV full width at half maximum (FWHM) is 39 $\AA$ and the integral of the $\mathrm{CV}$ profile gives a value of $1 \times 10^{12} \mathrm{~cm}^{-2}$ for the areal hole concentration ${ }^{5}$. The solid line is a theoretical calculation, where the Poisson and Schrödinger equations were solved self-consistently 
within the Hartree approximation, assuming the impurities follow a gaussian distribution of width equal to $5 \AA$, in the same way as it has been previously done for $\mathrm{Si}$ delta-doped $\mathrm{InP}^{6,7}$. A single heavy hole subband was taken into account in the calculations, given that the Fermi energy in the structures considered was always smaller than the energy separation between decoupled heavy and light hole subbands. The solid line in Fig. 1 shows the theoretical CV FWHM ( in units of the heavy hole Bohr radius, $\mathrm{a}_{B}=11.4 \AA$ ) as a function of the two dimensional hole concentration (in units of $\left.\mathrm{a}_{B}^{-2}\right)$ for an atomic $\mathrm{C}$ localization of $5 \AA, \mathrm{P}_{2 D}$. The triangles represent the samples grown for this work, while the other data points refer to results from the GaAs literature $^{8-11}$. Points above the solid line correspond to a confinement over a region wider than $5 \AA$.

In order to obtain such narrow confinement of the $\mathrm{C}$ atoms and a controlled high doping level, around $1 \mathrm{x}$ $10^{12} \mathrm{~cm}^{-2}$, several C-doped layers were grown using different $\mathrm{CBr}_{4}$ fluxes and various V/III. Fig. 2 shows [P] as a function of the $\mathrm{CBr}_{4}$ flux for these samples. The $\mathrm{V} / \mathrm{III}$ values used are shown in the inset. The solid symbols correspond to delta layers while the open ones correspond to bulk-doping. The points on the $\mathrm{Y}$ axis, are the residual doping levels achieved for the different $\mathrm{V} / \mathrm{III}$ values, meaning $\mathrm{CBr}_{4}$ flux equal to zero.

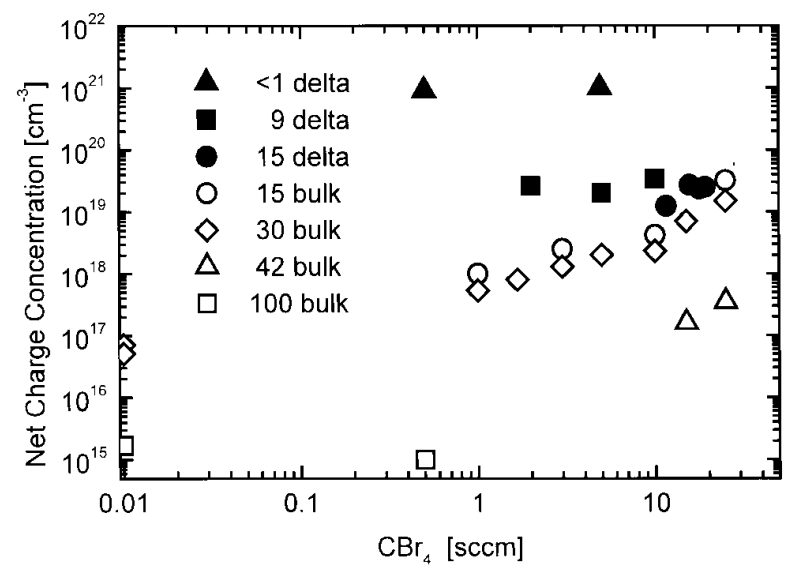

Figure 2. Net charge concentration as a function of the $\mathrm{CBr}_{4}$ flux. The solid symbols correspond to delta-doped layers and the open ones to bulk-doped layers. The V to III fluxes ratio used for the growth of the different samples are indicated in the figure.

One of the highlights of Fig. 2 are the extremely high doping levels achieved for V/III values below 10 due to the many sites $\mathrm{V}$ available for $\mathrm{C}$ atoms. However, as the $\mathrm{CBr}_{4}$ flux increases, little or no changes in the doping level is observed, meaning that one has no real control over the doping. With such low V/III, not only $\mathrm{C}$ from the $\mathrm{CBr}_{4}$ is incorporated but also from the TMGa and TMAl. This is the intrinsic doping regime. On the other extreme, for high V/III, only low or moderate doping levels are achieved. In the case of
$\mathrm{V} / \mathrm{III}=100$, the $\mathrm{C}$ residual doping is the lowest. Therefore, this ratio was chosen for the high $\mathrm{AsH}_{3}$ flux used to grow the undoped parts of the AlGaAs layers.

Comparing the delta-doped samples with the bulkdoped ones for V/III equal to 15 in Fig. 2, one observes the same behavior of $[\mathrm{P}]$ with the $\mathrm{CBr}_{4}$. For the required doping levels for the nipi amplitude modulators and other devices ${ }^{4,6,12},\left[1 \times 10^{18} \mathrm{~cm}^{-3}, 5 \times 10^{19} \mathrm{~cm}^{-3}\right]$, a very narrow range of V/III can be used. For the growth rate and $\mathrm{T}_{g}$ used in this investigation this interval is 15 to 30, as depicted in Fig. 2. In other words, one should keep in mind that despite its advantages, the $\mathrm{C}$ doping of AlGaAs leaves little flexibility in terms of growth conditions for some device applications.

Bulk GaAs layers delta-doped with Si were grown to optimize the impurity spatial distribution. C-V profiles were measured and typical FWHM of $65 \AA$ were observed for a doping level of $2.0 \times 10^{12} \mathrm{~cm}^{-2}$. This value is $20 \%$ below the theoretical resolution predicted in references 5 and 7 , implying in an impurity atomic localization of less than $10 \AA$, comparable to the best previously reported results ${ }^{5,8}$.

GaAs/AlGaAs MQW samples were then grown containing either $n$-type doping in the QW or $p$-type doping in the barrier in order to calibrate the electron and hole concentrations. The measured free electron concentration per doping plane, $N$, as a function of the silane flux for $100 \AA$ thick GaAs QWs showed a linear behavior for a $\mathrm{SiH}_{4}$ flux between 0.1 and $3.5 \mathrm{sccm}$. The mobility of the samples at RT is reduced as the doping level increases, as expected. Essentially no changes in mobility occur when the measurement temperature is lowered to $77 \mathrm{~K}$, because the mobility is limited by ionized impurities. It should be noted that the $n$-doping plane introduces a V-shaped potential well for electrons in the GaAs QW, so the free electrons are localized in the same region as the ionized $\mathrm{Si}$ atoms. The same types of measurements were performed in MQWs containing only $p$-type delta-doping in the center of the barriers. The two external barriers have half the doping level of the internal ones. The free hole concentration per doping plane, $P$, as a function of the $\mathrm{CBr}_{4}$ flux for the range between 2 and $20 \mathrm{sccm}$, also shows a linear behavior. The RT mobility of the $\mathrm{C}$ doped samples however is independent of the $\mathrm{CBr}_{4}$ flux. In this case, the $p$-type doping planes are in the barriers where they introduce an inverted $\mathrm{V}$ shaped potential, nevertheless part of the hole density moves into the QWs, staying far from the ionized impurities. This point will be re-addressed and further discussed. When no QWs are present, as in AlGaAs bulk material having a single C-doping plane, the mobility is lower because the holes remain close to the impurity plane. In addition, if the Hall measurement temperature is lowered to 77 $\mathrm{K}$, contrary to the Si delta-doped MQWs, the mobility increases indicating that it is limited by phonons and not by ionized impurity scattering. 
Having obtained the optimized growth conditions for the MQWs containing either types of doping, the nipi-MQW samples were grown using the calibration previuosly made with the purpose of obtaining essentially intrinsic material where $P$ and $N$ should be equal. However, the Hall measurements showed that the net free carrier concentration was heavily $n$-type. Assuming that the atomic incorporation is unchanged, either the electrical activity of the $p$-doping is reduced in the presence of Si atoms in the well or that of $n$-doping is improved in the presence of $\mathrm{C}$ atoms in the barriers. In trying to understand this effect, nipi-MQW samples with different silane fluxes were grown and the net free carrier concentration measured by Hall effect. Subtracting these values from those predicted using the previously obtained calibration curves, one obtains the concentration of "lost" free holes which is graphed in Fig. 3 as a function of the silane flux. One clearly sees that the concentration of "lost" free holes increases with the $\mathrm{SiH}_{4}$ flux. Moreover, the rate of increase is the same for the two different $\mathrm{CBr}_{4}$ fluxes, if a linear dependence is assumed, as depicted by the two solid lines in Fig. 3. In addition, extrapolating for zero silane flux, one finds that the density of holes lost for the undoped QWs increases with the $\mathrm{CBr}_{4}$ flux. In the case of zero $\mathrm{SiH}_{4}$ flux, the difference between the $\mathrm{C}$ atomic concentration, measured by SIMS, and the free hole concentration, measured by Hall, is in full agreement with the density of "lost" holes, shown in Fig. 3.

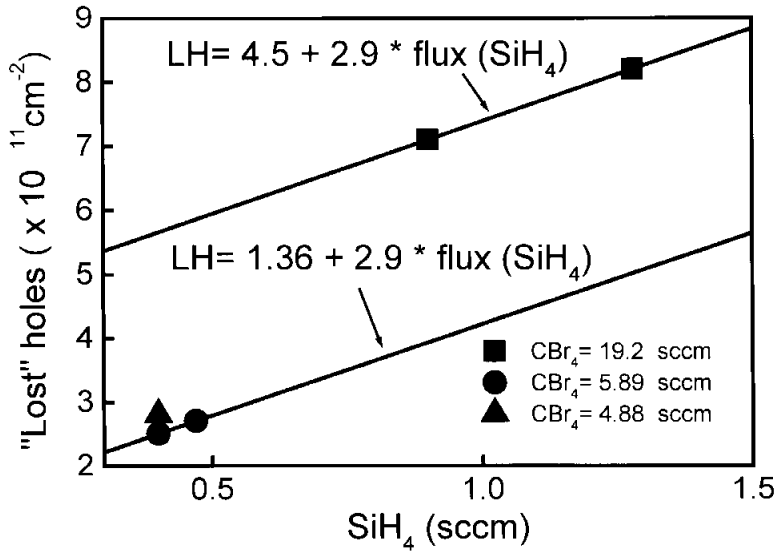

Figure 3. "Lost" free holes as a function of the $\mathrm{SiH}_{4}$ flux. These values are obtained from the subtraction of the measured net free carrier concentration for the MQW structures containing an nipi $\delta$-doping superlattice (nipi-MQW) from that predicted by the calibration.

The increase in "lost" holes with the silane flux can be understood as follows. The solid lines in Fig. 4 show a scheme of the potential for the nipi-MQW structure, while the dashed and solid lines correspond to the different hole probability densities, $F(z) * \mathrm{x} F(z)$, as labeled. With the presence of a plane of ionized Si atoms the QW is, in fact, divided into two half QWs. In the QW valence band the hole probability density corresponding to the fundamental energy level will be peaked closer to the interfaces, while in the case of an undoped QW, it will have its maximum in the center. In addition, the larger the $\mathrm{Si}$ atomic concentration, the deeper the $\mathrm{V}$-shaped potential in the QW, and consequently, the closer to the interface the hole probability density peak is. Thus, in the presence of hole traps in the interface, the introduction of an $n$-type doping plane in the QW will certainly lower the efficiency of the $p$-doping in the barriers. Moreover, this efficiency is directly dependent on the $n$-type impurity concentration. Further support for the substantial presence of holes close to the interfaces is encountered in the PL spectra.

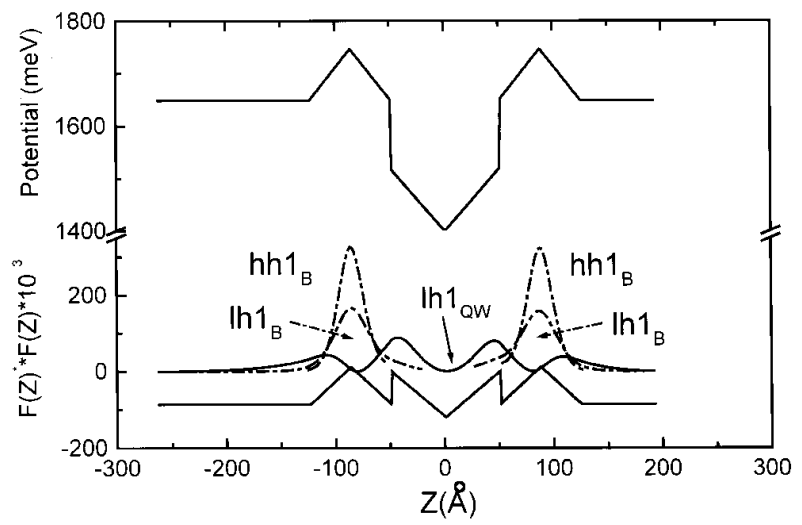

Figure 4. Scheme of the potential for a QW $n$ delta-doped in the center with $p$ delta-doped barriers. Also included are the hole probability densities for the different energy levels. The solid lines correspond to the hole probability density inside the GaAs QW while the dashed lines correspond to the hole probability densities in the inverted V-shaped potential in the AlGaAs barriers. The subscripts B and QW refer to the barriers and the quantum well, respectively.

The RT PL spectra of the nipi-MQW samples showed no emission below the fundamental gap. The near-edge optical transition was a few meV above that obtained for a sample with exactly the same MQW structure but undoped, which was used as reference. These results indicate that the introduction of the delta-doping superlattice should not harm the optical properties of the MQW structure for use in amplitude modulators.

PL measurements as a function of temperature however, revealed differences in the optical properties between the doped and the reference samples. Fig. 5 shows how the PL peak energy changed with temperature for both samples. The lines are theoretical calculations to be described below. The reference sample, represented by solid circles, behaves as should the optical transition corresponding to the first electron-heavy hole transition in the $\mathrm{QW},\left(\mathrm{e} 1_{Q W}-\mathrm{hh} 1_{Q W}\right)$, as depicted by the dashed line in Fig. 5. For the doped sample, the PL spectra as a function of temperature show that there exist two different emissions, one at low temperatures which reduces in intensity as the temperature is raised 
and another one at higher energy which is only observable at higher temperatures. At temperatures above 90 $\mathrm{K}$, this second emission dominates the spectra. In order to better understand these results, calculations of the temperature dependent electronic structure, within the full k.p Hamiltonian model were performed, where a triangular potential model (Fig. 4) was used to simulate the localization of the carriers in each doped layer, at their nominal densities. At low temperatures (below $50 \mathrm{~K}$ ), the experimental PL results are best described by the transition between the first QW electron and the first barrier heavy-hole levels $\left(e 1_{Q W}-\mathrm{hh} 1_{B}\right)$; the corresponding hole probability densities of the different energy levels involved are depicted in Fig. 4. Such transition is spatially indirect. At temperatures above $90 \mathrm{~K}$, the experimental results follow the temperature behavior of the transition between the first QW electron and first barrier light hole levels $\left(e 1_{Q W}-\mathrm{lh} 1_{Q W}\right)$ which is direct and only a few meV below the $\left(e 1_{Q W^{-}}\right.$ $\left.h \mathrm{hh} 1_{Q W}\right)$ transition of the equivalent undoped sample, as illustrated in Fig. 5. Thus, around $90 \mathrm{~K}$ the PL emission suffers a transition from indirect to direct. Experimental support for this change in nature is found in the results of PL as a function of excitation power (P). The PL peak energy of the emission at low temperatures is blue-shifted with increasing $P$, which is the standard behavior for spatially indirect transitions ${ }^{13}$, while that of the emission at high temperatures is unaffected by $\mathrm{P}$, indicating a direct transition. Since at RT the $\left(\mathrm{e} 1_{Q W}-\mathrm{lh} 1_{Q W}\right)$ transition dominates, one concludes that the $l h 1_{Q W}$ level, in the half QW, is indeed populated, demonstrating that holes are transferred from the barrier into the QWs. As a consequence, part of the transferred holes, being close to the interface as shown by the probability densities in Fig. 4, are most likely trapped by interface states.

\section{Summary}

GaAs/AlGaAs MQW structures containing an nipi delta-doped superlattice were investigated in order to evaluate their potential for use in high performance amplitude modulators based on the QCSE. First, it has been shown that it is possible to obtain suitable C deltadoped AlGaAs layers even though little flexibility is left in choosing the growth conditions for a controlled $\mathrm{C}$ doping of AlGaAs layers. Second, it has been demonstrated that to achieve the required balance between the $n$ and $p$ type doping levels one should consider the existence of hole traps at the interfaces whose occupancy depends on the QW n-doping level. Finally, PL measurements have shown that at RT only the nearedge emission is observed, and it is only a few $\mathrm{meV}$ below the fundamental transition of an equivalent undoped QW, not impairing, in principle, the use of such structures in the fabrication of amplitude modulators.

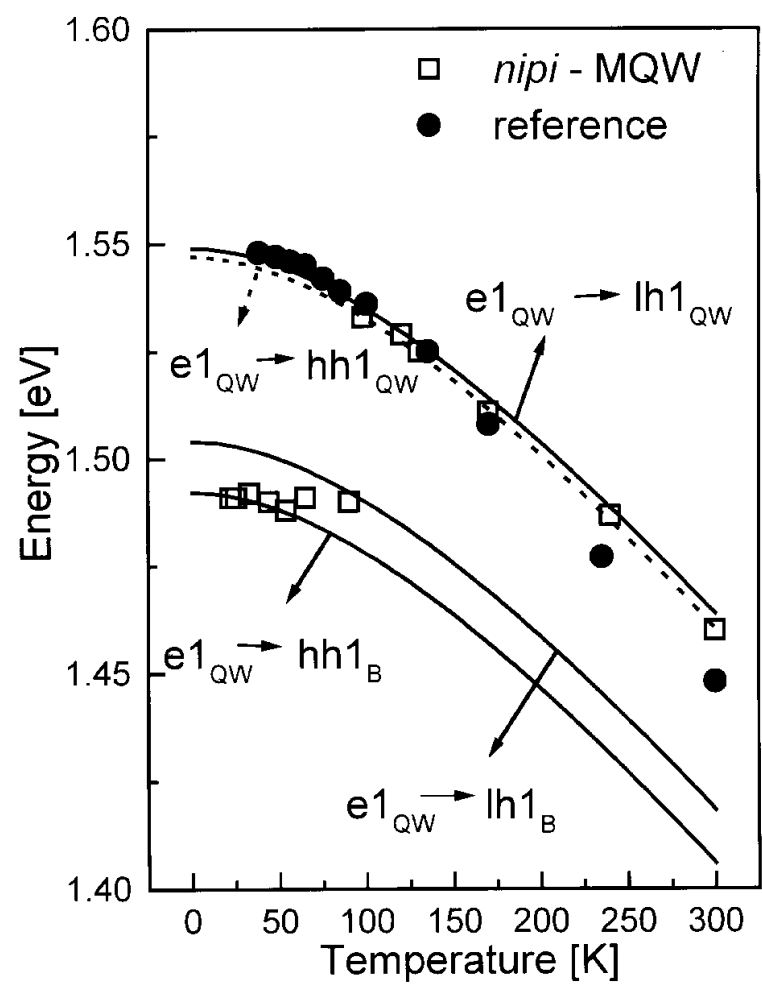

Figure 5. PL peak energy as a function of temperature. Solid circles represent the measurements for the reference sample whose behavior follow the electron-heavy hole transitions $\left(\mathrm{e} 1_{Q W} \prod \mathrm{hh} 1_{Q W}\right)$, represented by the dotted line. Open squares are obtained from the measurements for a MQW structure containing an nipi $\delta$-doping superlattice (nipi-MQW). At high temperatures (above $90 \mathrm{~K}$ ) the experimental points are best described by the $\left(\mathrm{e} 1_{Q W} \quad \prod \operatorname{lh} 1_{Q W}\right)$ transition, which involves electron and hole levels inside the GaAs QW (direct transition). At low temperatures, the experimental points are best described by the $\left(\mathrm{e} 1_{Q W} \prod\right.$ $\left.\mathrm{hh} 1_{B}\right)$ transition from the first electron level in the GaAs QW to the first heavy hole level in the AlGaAs barrier (indirect transition). At intermediary temperatures (50-90K) the experimental points are described by the $\left(\mathrm{e} 1_{Q W} \prod\right.$ $\left.l \mathrm{lh} 1_{B}\right)$ indirect transition which involves the QW electron and the barrier light- hole levels. The probability densities for the holes, which participate in the emissions described above, are schematically shown in Fig. 4.

\section{Acknowledgements}

This work has been partially financed by CNPq, CAPES, FAPERJ, ERICSSON and FAPESP.

\section{References}

[1] M. K. Chin and W. S. C. Chang, IEEE J. Quantum Electron. 29, 2476 (1993)

[2] T. Ido, H. Sano, D. J. Moss e H. Inoue, J. Lightwave Technol. 14, 2324 (1996).

[3] G. Bastard, E. E. Mendez, L. L. Chang and L. Esaki, Phys. Rev. B 28, 3241 (1983). 
[4] W. Batty and D. W. E. Allsopp, Electron. Lett., 29, 2066 (1993).

[5] E. F. Schubert in Semicondutores and Semimetals 40 , chapter 1, edited by A. C. Gossard, Academic Press, New York (1994).

[6] B. Yavich, P. L. Souza, M. P. Pires, A. B. Henriques, and L.C. D. Gonçalves, Semicon. Sci. and Technol. 12, 481 (1997).

[7] P. L. Souza in InP and Related Compounds: Materials, Applications and Devices, edited by M. O. Manasreh, chapter 6, (2000).

[8] B. R. Davidson, L. Hart J., R. C. Newman, T. B. Joyce T. J. Bullough and C. C. Button, J. of Mat. Sci.: Materials in Electronics 7, 355 (1996).

[9] T. Makimoto and N. Kobayashi, Jpn. J. Appl. Phys. 32, L1300 (1993).
[10] N. Kobayashi, T. Makimoto and Y. Horikishi, Appl. Phys. Lett. 50, 1435 (1987).

[11] M. B. Johnston, M. Gal, G. Li and C. Jagadish, J. Appl. Phys. 82, 5748 (1997).

[12] F. Brunner, T. Bergunde, E. Richter, P. Kurpas, S. Gramlich, I. Rechenberg, S. Kraus, M. Achouche, J. Würfl and M. Weyers, Proceedings of the EWMOVPE VIII, Prague-1999.

[13] Y. Gong, J. Mo, H. Yu. L. Wang and G. Xia, J. Crys. Gr. 209, 43, (2000).

[14] G. E. Höfler, H. J. Höfler, N. Holonyak, Jr. and K. C. Hsieh, J. Appl. Phys. 72, 5318 (1992).

[15] P. Abraham, M. A. Garcia Perez, T. Benyattou, G. Guillot, M. Sacolotti and X. Letartre, Semiconductor Science and Technology 10, 1585 (1995). 